\title{
Estimation of thermophysical properties of a large polar molecule and application to homogeneous nucleation of I-menthol
}

\author{
Carol Becker \\ Howard Reiss \\ Richard H. Heist \\ Fairfield University, rheist@fairfield.edu
}

Follow this and additional works at: https://digitalcommons.fairfield.edu/engineering-facultypubs (c) 1978 American Institute of Physics.

The final publisher PDF has been archived here with permission from the copyright holder. https://doi.org/10.1063/1.436216

\section{Peer Reviewed}

\section{Repository Citation}

Becker, Carol; Reiss, Howard; and Heist, Richard H., "Estimation of thermophysical properties of a large polar molecule and application to homogeneous nucleation of I-menthol" (1978). Engineering Faculty Publications. 150.

https://digitalcommons.fairfield.edu/engineering-facultypubs/150

\section{Published Citation}

Becker, C., Reiss, H., \& Heist, R. H. (1978). Estimation of thermophysical properties of a large polar molecule and application to homogeneous nucleation of I-menthol. The Journal of Chemical Physics, 68(8), 3585-3594. doi:10.1063/1.436216.

This item has been accepted for inclusion in DigitalCommons@Fairfield by an authorized administrator of DigitalCommons@Fairfield. It is brought to you by DigitalCommons@Fairfield with permission from the rightsholder(s) and is protected by copyright and/or related rights. You are free to use this item in any way that is permitted by the copyright and related rights legislation that applies to your use. For other uses, you need to obtain permission from the rights-holder(s) directly, unless additional rights are indicated by a Creative Commons license in the record and/or on the work itself. For more information, please contact digitalcommons@fairfield.edu. 


\title{
Estimation of thermophysical properties of a large polar molecule and application to homogeneous nucleation of I-menthol
}

\author{
Carol Becker and Howard Reiss \\ Department of Chemistry, University of California, Los Angeles, California 90024 \\ Richard H. Heist \\ Department of Chemical Engineering, University of Rochester, Rochester, New York 14627 \\ (Received 3 October 1977)
}

\begin{abstract}
The classical Becker-Doering theory has predicted to within $5 \%$ the critical supersaturations required for homogeneous nucleation for more than a dozen molecules. These successes have prompted us to investigate whether the classical theory can predict the experimental results for more extreme cases, i.e., high temperature measurements on large, polar molecules with hydrogen-bonding functional groups, like $l$-menthol. It is therefore the purpose of this paper (1) to report high temperature diffusion cloud chamber measurements on $l$-menthol vapor: (2) to present the thermophysical properties, data or calculated values. required for (a) the experiment, i.e., the mass and heat flux equations, (b) the theory, and (c) the film correction; and (3) to compare the predictions of the Becker-Doering theory with the experimental results. Critical supersaturations, measured over the temperature range $41-100^{\circ} \mathrm{C}$, are about $7 \%$ higher than those predicted by the theory. Applying a film correction to the raw data brings the experimental envelope to within $5 \%$ of the theoretical curve. On the basis of this success the classical theory can indeed be extended to include high temperatures and unique molecules. However, the experimental results lie above the theory curve, indicating that menthol may be somewhat associated in the vapor phase. Accurate experimental supersaturations can be obtained for a molecule even though its thermophysical properties are scarce. Except for the vapor pressure, all the properties of menthol used in the mass and heat flux equations are calculated by reliable theoretical or empirical estimation methods: Varying each property by an amount equal to the error inherent in the corresponding estimation method has an insignificant effect on the supersaturation profile in the chamber. In fact, neglecting the film correction is a larger source of experimental error than estimating all the properties (except the vapor pressure) required to solve the mass and heat flux equations. Since the vapor pressure is critical to the experimental results, one set of self-consistent vapor pressure data for menthol, measured over the range $30-175^{\circ} \mathrm{C}$, is needed. Given a more accurate vapor pressure expression, we can determine to what degree menthol is associated in the vapor.
\end{abstract}

\section{INTRODUCTION}

In spite of its approximations, the classical BeckerDoering theory has correctly predicted the critical supersaturations required for homogeneous nucleation for more than a dozen molecules. These include methanol and ethanol, ${ }^{1-3}$ the $n$-alkanes, ${ }^{4}$ water, ${ }^{5,6}$ the $n$-alkyl benzenes, ${ }^{7,8}$ and halogenated methane and ethane. ${ }^{7,9}$ These successes have prompted us to investigate whether the Becker-Doering (BD) theory can predict the experimental results for more extreme cases, i.e., high temperatures and unique molecules. Menthol (1-methyl-4isopropyl-3-cyclohexanol) meets these requirements. Unlike the other molecules studied, it is a solid at room temperature (melting point $=42.5^{\circ} \mathrm{C}$ ). This limits measurements to an unusually high temperature domain. In fact, the menthol experiment is the highest temperature nucleation study reported to date. Critical supersaturations are measured from $41^{\circ} \mathrm{C}$ (supercooled) to temperatures as high as $100^{\circ} \mathrm{C}$ where the onset of convection could become a problem.

Menthol is unique in that it is by far the largest alcohol studied in the cloud chamber. Its hydrogen-bonding hydroxyl group is responsible for a dipole moment (about 1.76 D in the vapor) ${ }^{10,11}$ comparable to that of water. In addition, naturally occurring $l$-menthol is the first optical isomer (specific rotation $\left.=-48^{\circ}\right)^{12}$ to be investigated in the cloud chamber. However, men- thol is unique for yet another reason-although it is an abundant substance ${ }^{13}$ its thermophysical properties are scarce. No data is available in the literature for any of the properties needed to analyze the raw cloud chamber data or compute the theory curve, except the molecular weight, the liquid density, and equilibrium vapor pressures above 2.8 torr $\left(73^{\circ} \mathrm{C}\right)$.

It is therefore the purpose of this paper (1) to report high temperature diffusion cloud chamber measurements on $l$-menthol vapor; (2) to present the thermophysical properties, data or calculated values, required for (a) the experiment (the mass and heat flux equations), (b) the $\mathrm{BD}$ theory, and (c) the film correction; and (3) to compare the predictions of the $\mathrm{BD}$ theory for this large polar molecule with the experimental results.

\section{METHODOLOGY}

\section{A. Chamber}

Since a detailed description of the chamber has been given elsewhere, ${ }^{3,4}$ only the special aspects of our design are mentioned here. The chamber is represented by the schematic diagram shown in Fig. 1. The plates are solid copper disks, $20.3 \mathrm{~cm}$ in diameter and 2.4 $\mathrm{cm}$ thick. There are two small thermocouple holes near the center of the bottom plate, drilled to within $0.3 \mathrm{~cm}$ of its flat upper surface. A thermocouple (dotted line) placed in each hole makes thermal contact with the plate 


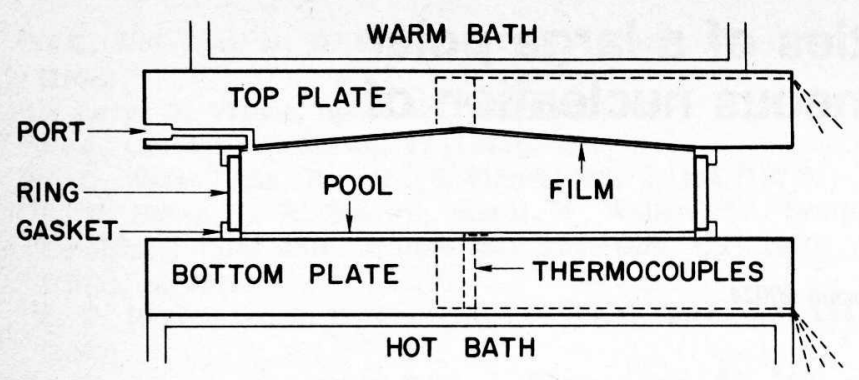

FIG. 1. Schematic diagram of the diffusion cloud chamber used in the menthol experiments.

via the low temperature solder which fills the hole. A third thermocouple hole at the center is drilled through the plate. A fluorogold plug (with a thermocouple wire threaded through it) is inserted into the hole and compressed by a screw device until its tapered end protrudes just above the surface of the plate, producing a vacuum tight seal. The top of the thermocouple bead is positioned $2.0 \mathrm{~mm}$ above the plate surface during chamber operation.

The top plate is machined on its lower surface to have a $1^{\circ}$ bevel so that condensate can drain off to the sides of the chamber. Like the bottom plate it has two thermocouple holes near the center drilled to within $0.3 \mathrm{~cm}$ of its beveled surface, but, in addition, one carrier gas port just inside the outer edge of the working surface.

The five copper-constantan thermocouples are calibrated over the temperature range -50 to $190^{\circ} \mathrm{C}$ against a platinum resistance thermometer standardized at the National Bureau of Standards.

The plates are separated by a Pyrex ring which forms the walls of the chamber. The ring is $2.35 \mathrm{~cm}$ high with an average inside diameter of $14.3 \mathrm{~cm}$ and an average wall thickness of only $0.36 \mathrm{~cm}$. Two nichrome heater wires (not shown) of varying resistances, i.e., 30 and 28 gage, are connected in series and wrapped around the ring. These supply the $17-29 \mathrm{~W}$ required to just clear the walls of condensate. L-shaped viton gaskets $0.26 \mathrm{~cm}$ thick are used as seals between the plates and the edges of the Pyrex ring. For this chamber the height measured at half-radius is $2.68 \mathrm{~cm}$, giving a diameter : height ratio of $5.3: 1$.

The plates are maintained at their respective temperatures by surface contact with self-contained constant temperature baths. The bottom plate rests on a bath $20.3 \mathrm{~cm}$ in diameter (the same diameter as the plate) which is connected by flexible stainless steel hoses to a Lauda NBS-HT constant temperature circulator. With "Crisco" oil as the circulating fluid, this, heating unit maintains the plate at temperatures constant to better than $0.1{ }^{\circ} \mathrm{C}$ over the temperature range $100-175^{\circ} \mathrm{C}$. A similar heating unit placed on the top plate maintains constant plate temperatures from $30-85^{\circ} \mathrm{C}$ with water as the circulating fluid.

Nucleation is easily observed by focusing the light from a $650 \mathrm{~W}$ tungsten-halide lamp into the chamber.
In order to eliminate ion-induced nucleation, ions are swept out by an electric field of $112 \mathrm{~V} \mathrm{~cm}^{-1}$ applied across the plates during observation.

The reliability of the chamber has been verified by performing homogeneous nucleation experiments on $n$-butylbenzene. The results agree with published data to within $5 \% .^{8}$

\section{B. Experiment}

One experimental point consists of three measurements: the temperature of the pool surface $\left(T_{0}\right)$, the temperature of the top plate $\left(T_{1}\right)$, and the total pressure in the chamber $\left(P_{T}\right)$. The experiment is performed as follows:

Menthol crystals ( $98 \%$ pure, MCB; Eastman), sufficient to form a liquid pool $2.0 \mathrm{~mm}$ deep, are placed on the bottom plate and the chamber sealed. The chamber is carefully evacuated and backfilled with helium (99.995\% pure) several times while the circulators come to temperature. After the hot circulating fluids are admitted into the baths, the wall heat is gradually increased to keep the walls just free of condensate. As soon as the plates are at temperature, the chamber is illuminated and any heavy nucleation can easily be observed. An electric field is applied across the plates and the temperature of the bottom plate decreased several tenths of a degree until a slow, steady rain is observed which falls straight downward in all parts of the chamber at a rate of $1 \mathrm{drop} \mathrm{cm}^{-3} \mathrm{sec}^{-1}$. The chamber is left alone for about $40 \mathrm{~min}$ to ensure complete equilibrium. If, at the end of this time, the rate remains unchanged at a steady 1 drop $\mathrm{cm}^{-3} \mathrm{sec}^{-1}$, the reading from the pool thermocouple is displayed on a pen strip chart and the average recorded as $T_{0}$. The two top plate thermocouple readings, which always agree to within $0.05^{\circ} \mathrm{C}$, are averaged and taken as $T_{1} . \quad P_{T}$ is read from a mercury manometer and a mercury correction applied.

To obtain the next experimental point the temperature of each plate is changed by a few degrees, the pressure adjusted, and the sequence repeated. This is continued until the entire accessible temperature range has been investigated.

\section{Mass and heat flux equations}

Assuming plane parallel diffusion, the equations for the mass and heat fluxes in the chamber are

$$
\begin{aligned}
& \frac{d P}{d z}=\frac{\alpha P\left(P-P_{T}\right)}{T P_{T}} \frac{d T}{d z}+\frac{L\left(P-P_{T}\right)}{T^{s} D_{v g}^{0}}, \\
& \frac{d T}{d z}=\lambda_{v g}^{-1}\left\{-q+L\left[H_{v}(T)-H_{l}\left(T_{1}\right)\right]+\frac{\alpha R T L\left(P_{T}-P\right)}{P_{T}}\right\},
\end{aligned}
$$

where $P$ is the partial pressure of the vapor, $T$ the absolute temperature, and $R$ the gas constant. $z$ is a reduced chamber height $z=Z / h, h$ being the actual chamber height, so that $0 \leqslant z \leqslant 1$. $L$ is a reduced molar flux $L=L_{v} h$, where $L_{v}$ is the molar flux of the vapor, and $q$ is a reduced heat flux $q=Q h$. $\alpha$ is a thermal diffusion ratio defined as

$$
\alpha=k_{T} / X_{v} X_{g},
$$


where $k_{T}$ is the thermal diffusion factor, and $X_{v}=P / P_{T}$, the mole fraction of the vapor. $D_{v g}^{0}$ and the exponent $s$ are constants related to the binary diffusion coefficient $D_{v g}$ in the following way:

$$
D_{v g}=D_{v g}^{0} R T^{s+1} / P_{T} \text {. }
$$

$\lambda_{v g}$ is the thermal conductivity of the vapor-carrier gas mixture. Choosing $T_{1}$ as a reference temperature, we can express $H_{v}(T)$, the enthalpy of the vapor at any temperature $T$, as

$$
H_{v}(T)=H_{v}\left(T_{1}\right)+\int_{T_{1}}^{T} C_{\phi_{v}} d T,
$$

where $C_{\phi_{v}}$ is the molar heat capacity of the vapor. Finally, $H_{l}\left(T_{1}\right)$ is the enthalpy of the condensed liquid at $T_{1}$.

The quantity $H_{v}(T)-H_{l}\left(T_{1}\right)$ in Eq. (2) can be evaluated by making use of the relation

$$
H_{v}(T)-H_{l}\left(T_{1}\right)=H_{v}(T)-H_{v}\left(T_{1}\right)+H_{v}\left(T_{1}\right)-H_{l}\left(T_{1}\right),
$$

where $H_{v}\left(T_{1}\right)-H_{l}\left(T_{1}\right)=\Delta H_{\text {vap }}\left(T_{1}\right)$, the enthalpy of vapor ization at $T_{1}$. Substituting the definition given in Eq. (5) for $H_{v}(T)-H_{v}\left(T_{1}\right)$ into Eq. (6), we can restate the lefthand side of Eq. (6) as

$$
H_{v}(T)-H_{l}\left(T_{1}\right)=\int_{T_{1}}^{T} C_{p_{v}} d T+\Delta H_{\mathrm{vap}}\left(T_{1}\right)
$$

Equation (2) then becomes

$$
\frac{d T}{d z}=\lambda_{v B}^{-1}\left\{-q+L\left[\int_{T_{1}}^{T} C_{D_{v}} d T+\Delta H_{\mathrm{vap}}\left(T_{1}\right)\right]+\frac{\alpha R T L\left(P_{T}-P\right)}{P_{T}}\right\} .
$$

Using a method formulated by $\mathrm{Katz},{ }^{3}$ Eqs. (1) and (8) are solved numerically for the partial pressure and temperature profiles in the chamber subject to the following boundary conditions: At the bottom of the chamber, where $z=0, T(0)=T_{0}$, and $P(0)=P_{e}\left(T_{0}\right)=P_{0}$; at the top, where $z=1, T(1)=T_{1}$, and $P(1)=P_{e}\left(T_{1}\right)=P_{1}$. Here $P_{e}$ refers to the equilibrium vapor pressure of the working fluid while $P$ is the actual pressure of the vapor.

The approximations $P(0)=P_{e}\left(T_{0}\right)$ and $P_{1}=P_{e}\left(T_{1}\right)$ can be made only when the flux ratio $L_{v}\left[P /(2 \pi M R T)^{1 / 2}\right]^{-1}$ is very small, where $P /(2 \pi M R T)^{1 / 2}$ represents the equilibrium flux of molecules, and $M$ is the molecular weight of the working fluid. ${ }^{3}$ These approximations are valid for menthol since the flux ratio is typically $10^{-5}$ at the evaporating (pool) surface and $10^{-3}$ at the condensing (film) surface.

The thermophysical properties needed to solve Eqs. (1) and (8) and analyze the raw cloud chamber data are, for the working fluid, (1) the equilibrium vapor pressure, (2) the vapor viscosity, (3) the vapor heat capacity, (4) the vapor thermal conductivity, (5) the enthalpy of vaporization at $T_{1}$, and (6) the molecular weight. Of these, only the molecular weight and vapor pressures above 2.8 torr are available in the literature for menthol.

For the vapor-carrier gas mixture (1) the thermal conductivity, (2) the thermal diffusion ratio, (3) the binary diffusion coefficient, and (4) $s$, the binary diffusion coefficient temperature dependence, are required. All of these must be calculated for menthol-helium or approximated by analogy with values for other mixtures.

For the carrier gas alone the only properties needed are (1) the gas viscosity $\left(\eta_{g}\right)$, (2) the gas thermal conductivity $\left(\lambda_{g}\right)$, and (3) the molecular weight. Reliable data for these three have been published for helium. The remainder of this section will be devoted to a brief description of the methods used to estimate the properties of the working fluid unavailable in the literature.

For menthol the vapor viscosity $\left(\eta_{v}\right)$, required over the range $30-175^{\circ} \mathrm{C}$, is calculated in three different ways. Each set of calculated values, which agree to within $5 \%$ at any given $T$, is fit to the two-constant Sutherland equation ${ }^{14}$

$$
\eta_{v}=K T^{3 / 2} /(T+D) \text {. }
$$

The resulting values of $K$, and likewise the values of $D$, are averaged to obtain the Sutherland constants for menthol presented in Table I. The three estimation methods are discussed in greater detail in the Appendix.

The ideal gas heat capacity $\left(C_{p}\right)$ is calculated by $\mathrm{Ri}-$ hani and Doraiswamy's method of group contributions, which gives an average error of $4 \% \cdot{ }^{15}$ Once $\eta_{v}$ and $c_{p}$ are known, a simple kinetic-theory result, such as the Eucken equation, can be used to estimate the vapor thermal conductivity $\left(\lambda_{v}\right)^{16}$

$$
\lambda_{v}=\left(\eta_{v} / M\right)\left(C_{v}+9 R / 4\right),
$$

where $C_{v}$, the molar heat capacity at constant volume, is taken as $C_{p}-R$. For the Eucken theory [Eq. (10)] the average error in $\lambda_{v}$ is $16 \%$.

The enthalpy of vaporization $\left(\Delta H_{v}\right)$ is estimated by the Watson correlation between $\Delta H_{v}$ and temperature ${ }^{17}$

$$
\Delta H_{v_{2}}=\Delta H_{v_{1}}\left[\left(1-T_{r_{2}}\right) /\left(1-T_{r_{1}}\right)\right]^{0.38} \text {. }
$$

Here, subscripts 1 and 2 refer to temperatures 1 and 2, respectively; and the reduced temperature $T_{r}$ has the usual definition $T_{r}=T / T_{c}$, where $T_{c}$ is the critical temperature. In calculating $\Delta H_{v_{2}}$ for menthol we choose the enthalpy of vaporization at the boiling point $\left(\Delta H_{\mathbf{v a p}_{b}}\right)$ as $\Delta H_{v_{1}}$, the reference enthalpy in Eq. (11), because $\Delta H_{\mathbf{v a p}_{b}}$ can be calculated accurately from the critical constants $^{18}$ using the Riedel empirical method ${ }^{19}$

$$
\Delta H_{\mathrm{vad}_{b}}=T_{b}\left(5 \log P_{c}-2.17\right) /\left(0.930-T_{b_{r}}\right) \text {, }
$$

where $P_{c}$ is the critical pressure and $T_{b}$ is the normal boiling point. The constants $\Delta H_{v_{a p}}, T_{b}$, and $T_{c}$ used in Eq. (11) to estimate $\Delta H_{\mathrm{vav}}\left(T_{1}\right)$ for menthol are listed in Table I. With the use of estimated values for $\Delta H_{\mathrm{vap}_{b}}$ and $T_{c}$, the average error in Eq. (11) is 6\%.

An average error has been quoted for each of the estimation methods described above: $\eta_{v}, 8 \% ; C_{p_{v}}, 4 \%$; $\lambda_{v}, 16 \%$; and $\Delta H_{\text {vap }}\left(T_{1}\right), 6 \%$. Previous workers have shown that changing $\eta_{v}$ by $10 \%$, or $C_{p_{v}}$ by $15 \%$, has a completely negligible effect on the supersaturation profile in the chamber. ${ }^{4,9}$ We find that a $20 \%$ change in $\lambda_{v}$ changes the peak supersaturations by only $0.3 \%$. Therefore, a large uncertainty in the calculated thermal conductivity is an insignificant source of error. The 
TABLE I. Thermophysical properties required to solve the mass and heat flux equations are indicated by an e; to calculate the theory by a t; and to make the film correction by an fc. Symbols have the following meaning: $P_{e}$, equilibrium vapor pressure; $\eta$, viscosity; $C_{p}$, heat capacity at constant pressure; $\lambda$, thermal conductivity; $\Delta H_{\text {vap }}$, enthalpy of vaporization; $\sigma$, surface tension; $\rho$, density; $\Delta S^{*}$, Everett entropy of vaporization; $w$, Watson expansion factor; $\alpha$, thermal diffusion ratio; $D$, binary diffusion coefficient; and $s$, binary diffusion coefficient temperature dependence. Subscripts have the following meaning: $v$, vapor; $v g$, vapor-carrier gas; $g$, carrier gas; and $l$, liquid. Expressions are valid over the following temperature ranges: experiment, 30$175^{\circ} \mathrm{C}$; theory, $35-125^{\circ} \mathrm{C}$; and film correction, $30-85^{\circ} \mathrm{C}$. Temperatures are in ${ }^{\circ} \mathrm{K}$ unless otherwise specified.

\begin{tabular}{|c|c|c|}
\hline & Used in & Reference \\
\hline \multicolumn{3}{|l|}{ Menthol } \\
\hline $\log _{10} P_{e}($ torr $)=6.1102-\frac{1093.1}{T-155.28}$ & $e, t$ & 21,22 \\
\hline$\eta_{v}(c P)=\frac{\left(7.8226 \times 10^{-4}\right) T^{1.5}}{T+397.98}$ & e & $16,18,46-48$ \\
\hline$C_{\mathrm{A}_{2}}\left(\mathrm{cal} \mathrm{mole} \mathrm{m}^{-1} \mathrm{o}^{-1}\right)=-17.963+0.28507 T+\left(1.7426 \times 10^{-4}\right) T^{2}$ & $\mathrm{e}$ & 15 \\
\hline$\lambda_{v}\left(\mathrm{cal} \mathrm{cm} \mathrm{cm}^{-1} \sec ^{-1}{ }^{\circ} \mathrm{K}^{-1}\right)=\frac{\eta_{v}}{M}\left(C_{p v}+2.48\right)$ & $\mathrm{e}$ & 16 \\
\hline where $M\left(\mathrm{~g} \mathrm{~mole}^{-1}\right)=156.27$ & $e, t, f c$ & \\
\hline$\Delta H_{\text {vad }}\left(T_{1}\right)\left(\right.$ cal mole $\left.^{-1}\right)=\Delta H_{\text {rapb }}\left(\frac{1-T_{r 1}}{1-T_{r_{b}}}\right)^{0.38}$ & $\mathrm{e}$ & 17 \\
\hline where $\Delta H_{\text {vab }_{b}}\left(\right.$ cal mole $\left.{ }^{-1}\right)=12915$ & $\mathrm{e}, \mathrm{fc}$ & 19 \\
\hline$T_{b}\left({ }^{\circ} \mathrm{C}\right)=212.0$ & & 12,21 \\
\hline$T_{c}\left({ }^{\circ} \mathrm{C}\right)=379.3$ & & 18 \\
\hline$\sigma\left(\right.$ dyne $\left.\mathrm{cm}^{-1}\right)=30.370-\left(8.1918 \times 10^{-2}\right)(T-273.16)$ & $\mathrm{t}$ & 32 \\
\hline$\rho_{l}\left(\mathrm{~g} \mathrm{~cm}^{-3}\right)=0.91557-\left(7.7554 \times 10^{-4}\right)(T-273.16)$ & $t$, fc & 30 \\
\hline $\ln \eta_{l_{1}}(P)=-23.933+(6946.7 / T)$ & fc & 34 \\
\hline $\ln \eta_{l_{2}}(P)=-17.383+(4785.1 / T)$ & fc & 35 \\
\hline $\begin{array}{l}\lambda_{l}\left(\mathrm{cal} \mathrm{cm}^{-1} \mathrm{sec}^{-1} \mathrm{o}^{-1}\right)=\frac{(88.0-4.94 G)\left(10^{-3}\right)}{\Delta S^{*}}\left(\frac{0.55}{T_{r}}\right)^{N} C_{p_{l}} p_{l}^{4 / 3} \\
\text { where } G=4 ; N=1 ;\end{array}$ & fc & 36 \\
\hline$\Delta S^{*}\left(\mathrm{cal} \mathrm{mole}{ }^{-1}{ }^{\circ} \mathrm{K}^{-1}\right)=25.48 ;$ & & 37 \\
\hline$C_{p_{l}}\left(\mathrm{cal} \mathrm{mole}{ }^{-1} \mathrm{~K}^{-1}\right)=\left(1.982 \times 10^{-3}\right) M / w^{2.8}$ & fc & $38-40$ \\
\hline \multicolumn{3}{|l|}{ Menthol-Helium } \\
\hline$\lambda_{v g}\left(\operatorname{cal~cm}^{-1} \sec ^{-1}{ }^{\circ} \mathrm{K}^{-1}\right)=\frac{\lambda_{v}}{1+A_{v g}\left(X_{g} / X_{v}\right)}+\frac{\lambda_{g}}{1+A_{g v}\left(X_{v} / X_{g}\right)}$ & e & 23 \\
\hline where $A_{v g}$ and $A_{g v}$ are Lindsay-Bromley parameters & e & 24 \\
\hline$\alpha=0.3$ & e & 3 \\
\hline$D_{\text {ug }}\left(\right.$ moles cm $\left.{ }^{-1} \mathrm{sec}^{-1}\right)=0.17505$ & e & 25 \\
\hline$s=0.75$ & $\mathrm{e}$ & 25 \\
\hline \multicolumn{3}{|l|}{ Helium } \\
\hline$\eta_{g}(c P)=\frac{\left(1.4808 \times 10^{-3}\right) T^{1.5}}{T+87.609}$ & e & 26 \\
\hline \multicolumn{3}{|l|}{$\lambda_{g}\left(\mathrm{cal} \mathrm{cm}^{-1} \mathrm{sec}^{-1}{ }^{\circ} \mathrm{K}^{-1}\right)=-6.7134 \times 10^{-5}+\left(2.4624 \times 10^{-6}\right) T$} \\
\hline$-\left(4.7622 \times 10^{-9}\right) T^{2}+\left(4.2650 \times 10^{-12}\right) T^{3}$ & $\mathrm{e}$ & 27 \\
\hline$M\left(\mathrm{~g} \mathrm{~mole}^{-1}\right)=4.0026$ & e & \\
\hline
\end{tabular}

solutions for $T$ and $P$ as a function of $z$ are independent of the magnitude of $\Delta H_{\mathrm{vap}}\left(T_{1}\right) .{ }^{4}$ However, changing $\Delta H_{\mathrm{vap}}\left(T_{1}\right)$ does affect one important quantity, the heat flux $(q)$ which is obtained along with the temperature profile from the solution of the heat flux equation [Eq. (8)]. We find that a $10 \%$ change in $\Delta H_{\text {vap }}\left(T_{1}\right)$ alters $q$ by $4.2 \%$, and a $4.2 \%$ change in $q$ has a negligible effect, i.e., $0.2 \%$, on the film corrected peak supersaturations. 
This insensitivity of experimental results to reasonable values of $\eta_{v}, C_{p_{v}}, \lambda_{v}$, and $\Delta H_{\text {vap }}\left(T_{1}\right)$ greatly increases the number of molecules that can be investigated in the diffusion cloud chamber. When data for these properties is unavailable, it can be estimated by the reliable theoretical or empirical methods used in this study. These methods have been thoroughly tested on large numbers of molecules of various types-nonpolar and polar molecules, hydrocarbons, and oxygen-containing compounds, to mention a few. Frequently, the error inherent in a particular estimation method is about the same magnitude as the experimental error inherent in actually measuring a property. For example, it is not uncommon for vapor viscosity data reported in the literature to be in error by $10 \% .^{8}$ In comparison, the average error quoted for estimated gas viscosities, obtained by the quasitheoretical methods discussed in the Appendix, is $8 \%$. Thus, even if most of the properties used in the mass and heat flux equations are unavailable in the literature, the supersaturation profiles can be accurately determined from estimated properties. This is true of all the properties required to analyze the raw cloud chamber data except one-the equilibrium vapor pressure of the working fluid. ${ }^{20}$

For menthol, Stull's compilation is the only extensive source of vapor pressure data in the literature. ${ }^{21} \mathrm{Va}$ por pressures range from 1 torr at $56^{\circ} \mathrm{C}$ to $1 \mathrm{~atm}$ at the boiling point $\left(212^{\circ} \mathrm{C}\right)$. We fit Stull's data to the Antoine form $\log _{10} P_{e}=A-B /(T-C)$ by the method of nonlinear least squares. The resulting Antoine coefficients $(A$ $=8.2598, B=2406.9, C=37.790$ ) reproduce the vapor pressures accurately, with an average error of $0.7 \%$. However, using this vapor pressure expression, we found, for most of the menthol data, a very large discrepancy $(\sim 40 \%)$ between theory and experiment. Only for the four highest temperature points, for which $T_{1}$ $>73^{\circ} \mathrm{C}$, did the theory predict the experimental results correctly. Additional vapor pressures in the range $31-73^{\circ} \mathrm{C}$ were needed for menthol. Fortunately, Ervin has measured 18 points in the temperature range of interest. $^{22}$ This unpublished data has provided the opportunity to obtain new values of the experimental supersaturations without having to actually repeat any of the experiments.

We determine the new Antoine coefficients by the method of nonlinear least squares, using all of Ervin's data and all of Stull's data combined, except Stull's point at $212^{\circ} \mathrm{C}$, which is beyond the temperature range of our experiments. After determining the coefficients for the combined data, we eliminate any points more than two standard deviations away from the calculated curve, and determine a new set of coefficients. This procedure is repeated until, for a given set of coefficients, all the observed data falls within two standard deviations of the calculated curve. The final coefficients, valid from $23.6-190.2{ }^{\circ} \mathrm{C}$, reproduce the observed vapor pressures with an average error of $4.2 \%$. They are listed in Table I along with the remaining thermophysical properties needed to analyze the raw cloud chamber data. ${ }^{23-27}$ Since these properties have been well documented elsewhere, ${ }^{8}$ the rest of this sec- tion will be devoted to a discussion of the theory and film correction.

\section{Theory}

The rate of nucleation $J\left(\mathrm{~cm}^{-1} \mathrm{sec}^{-3}\right)$ of a supersaturated vapor is given by the classical Becker-Doering equation $^{28,29}$

$$
J=\frac{a}{\rho_{l}}\left(\frac{2 N_{0}^{3} \sigma M}{\pi}\right)^{1 / 2}\left(\frac{S P_{e}}{R T}\right)^{2} \exp \left[\frac{-16 \pi N_{0}}{3(\ln S)^{2}}\left(\frac{M}{\rho_{l}}\right)^{2}\left(\frac{\sigma}{R T}\right)^{3}\right],
$$

where the condensation coefficient $a=1, \rho_{l}$ is the liquid density, $N_{0}$ is Avogadro's number, $\sigma$ is the surface tension of the liquid, and $S$ is the supersaturation $P / P_{e}$. We define the critical supersaturation $\left(S_{c}\right)$ as the supersaturation at the onset of homogeneous nucleation, i.e., at which the rate $J=1$. The variation of $S_{c}$ with $T$ can then be obtained by setting $J$ in Eq. (13) equal to unity and solving for $S_{c}$ at a given $T . S$ is a very insensitive function of $J$. For example, increasing $J$ by a factor of 10, a realistic experimental upper limit, causes a $5.2 \%$ increase in $S$ at low temperatures and a $2.6 \%$ increase at high temperatures. Therefore, to within a few percent the critical supersaturation predicted by the classical theory is well defined.

The thermophysical properties needed to calculate the theoretical variation of $S_{c}$ with $T$ are (1) the surface tension of the liquid, (2) the equilibrium vapor pressure, (3) the liquid density, and (4) the molecular weight. If we are to compare theory with experiment, these properties must be known over the entire range of the menthol measurements $35-125^{\circ} \mathrm{C}$. A set of liquid densities, valid from $40-180^{\circ} \mathrm{C}$, has been published. ${ }^{30}$. However, for the theory, by far the most important property is the surface tension. ${ }^{9}$ Only scattered surface tensions can be found in the literature for menthol. ${ }^{31}$ Fortunately, a set of unpublished data, measured by Dynes over the range $56-127^{\circ} \mathrm{C}$, has recently become available. ${ }^{32}$ Surface tensions from $35-56^{\circ} \mathrm{C}$ are obtained by linear extrapolation of Dynes' data, using the expression for $\sigma$ given in Table I.

The remaining properties required for the theory $\left(P_{e}\right.$ and $\left.M\right)$ are also used as input for the mass and heat flux equations. $P_{e}$ has been discussed in detail in Sec. II. C.

\section{E. Film correction}

$T_{1}$ is defined as the temperature at the surface of the liquid film condensed on the top plate. At low temperatures the error due to neglecting $\Delta T$, the temperature drop across the film, is negligible. ${ }^{4}$ As a result, a number of previous workers have approximated $T_{1}$ as the temperature of the top plate. ${ }^{9}$ However, $\Delta T$ is directly proportional to the heat flux, which may become sizeable at the high temperatures required for the menthol experiment. We therefore calculate a film correction for each experimental point.

The thickness of the film $t$ condensed on the conical top plate surface at a distance $r$ from the center of the chamber is given approximately by ${ }^{4}$

$$
t=\left[3 \eta_{l}(L / h) M r \cos ^{7} \phi_{0} / 2 \rho_{l}^{2} g \sin \phi_{0}\right]^{1 / 3},
$$


where $\eta_{l}$ is the viscosity of the liquid, $L$ is a reduced molar flux, $h$ is the actual chamber height, $g$ is the standard gravitational constant, and $\phi_{0}$ is the angle of the conical chamber top to the horizontal. Given a value of $t, \Delta T$ can be calculated by the equation ${ }^{4}$

$$
\Delta T=t(q / h) \lambda_{l},
$$

where $q$ is a reduced heat flux and $\lambda_{1}$ is the thermal conductivity of the liquid.

The liquid viscosity and the liquid thermal conductivity are required to make the film correction. Liquid viscosities are very sensitive to temperature. From the freezing point to slightly above the boiling point the best simple temperature-liquid viscosity function which has been proposed is the for $\mathrm{m}^{33}$

$$
\eta_{l}=A e^{B / T} .
$$

Here, $A$ and $B$ are positive, and $A$ is of the same order of magnitude as the gas viscosity. Two sets of liquid viscosity data, each spanning a different temperature range, were found in the literature for menthol. ${ }^{34,35}$ Each set is fit separately to Eq. (16) and the resulting expressions for $\eta_{l_{1}}$ and $\eta_{l_{2}}$ listed in Table I. $\eta_{l_{1}}$ should be used at temperatures below $56.8^{\circ} \mathrm{C} ; \eta_{l_{2}}$ covers the range $56.8-90.5{ }^{\circ} \mathrm{C}$.

The thermal conductivity of the liquid is calculated using the Robbins-Kingrea equation ${ }^{36,37}$ given in Table $I$, which requires $\Delta H_{\mathrm{vap}_{b}}, T_{b}, \rho_{l}$, and $C_{p_{l}}$, the molar heat capacity of the liquid. No liquid heat capacity data exists for menthol, but we estimate the heat capacity C $\left(\right.$ cal g $^{-1}{ }^{\circ} \mathrm{K}^{-1}$ ) by the Chow-Bright correlation ${ }^{38}$

$$
b=C w^{2 \cdot 8} \text {. }
$$

At $T_{r}<0.65$ the Watson expansion factor $w$ is satisfactorily expressed by ${ }^{39}$

$$
w=0.1745-0.0838 T_{r} .
$$

The empirical constant $b$ can be determined if $C$ is known at one temperature. We obtain this reference value of $C$ from Johnson and Huang's group contributions, a method designed for calculating heat capacities at one temperature only, $20^{\circ} \mathrm{C} .{ }^{40}$ Once the value of $b$ is known, the heat capacity at any other temperature within the Watson correlation range can be estimated from Eq. (17).

\section{ANALYSIS OF DATA}

The three measurables - the temperature of the pool surface $T_{0}$, the temperature of the top plate $T_{1}$, and the total pressure $P_{T}$-are presented for each experiment in Table II. Temperatures range from 31.9 (supercooled) to $175.0^{\circ} \mathrm{C}$, where the onset of convection could become a problem. Since helium has been reported to suppress convection at all temperatures, ${ }^{8}$ it is used as the carrier gas in these experiments. Two stability criteria, the pressure ratio $P_{T} / P_{0}$ and the density ratio $\rho_{1} / \rho_{0}$, are reported for each experiment, where $\rho_{1}$ and $\rho_{0}$ are the total density of the vapor-carrier gas mixture at $T_{1}$ and $T_{0}$, respectively. The minimum usable pressure ratio, for which results are independent of the amount of carrier gas used, is $2.5 .^{8}$ It can be seen that
TABLE II. Raw data for each experiment: $T_{0}$, the temperature of the pool surface; $T_{1}$, the temperature of the top plate; and $P_{T}$, the total pressure. Stability criteria: $P_{T} / P_{0}$, the pressure ratio; and $\rho_{1} / \rho_{0}$, the density ratio. $P_{0}$ is the equilibrium vapor pressure of the working fluid at $T_{0} ; \rho_{1}$ and $\rho_{0}$ are the total density of the vapor-carrier gas mixture at $T_{1}$ and $T_{0}$, respectively.

\begin{tabular}{llll|ll}
\hline \hline $\begin{array}{l}\text { Experiment } \\
\text { number }\end{array}$ & $T_{0}\left({ }^{\circ} \mathrm{K}\right)$ & $T_{1}\left({ }^{\circ} \mathrm{K}\right)$ & $P_{T}$ (torr) & $P_{T} / P_{0}$ & $\rho_{1} / \rho_{0}$ \\
\hline 1 & 376.21 & 305.07 & 552.4 & 38.015 & 0.619 \\
2 & 381.69 & 309.71 & 542.9 & 28.354 & 0.530 \\
3 & 384.03 & 311.68 & 532.3 & 24.816 & 0.491 \\
4 & 387.86 & 314.95 & 459.8 & 17.880 & 0.400 \\
5 & 390.50 & 317.20 & 539.7 & 18.592 & 0.411 \\
6 & 391.80 & 317.99 & 478.0 & 15.520 & 0.364 \\
7 & 394.26 & 319.45 & 551.4 & 16.050 & 0.373 \\
8 & 394.81 & 319.92 & 595.6 & 16.921 & 0.387 \\
9 & 396.08 & 322.16 & 488.3 & 13.124 & 0.324 \\
10 & 401.00 & 324.82 & 563.0 & 12.274 & 0.311 \\
11 & 401.76 & 326.23 & 542.6 & 11.462 & 0.296 \\
12 & 404.98 & 328.50 & 582.1 & 10.780 & 0.283 \\
13 & 407.68 & 329.63 & 567.8 & 9.439 & 0.257 \\
14 & 407.92 & 329.75 & 577.0 & 9.504 & 0.259 \\
15 & 410.16 & 332.62 & 542.8 & 8.189 & 0.232 \\
16 & 413.19 & 333.60 & 583.3 & 7.836 & 0.225 \\
17 & 412.86 & 333.69 & 521.9 & 7.099 & 0.208 \\
18 & 419.29 & 340.40 & 634.0 & 6.797 & 0.205 \\
19 & 429.51 & 346.23 & 613.6 & 4.612 & 0.154 \\
20 & 428.73 & 346.33 & 550.1 & 4.244 & 0.145 \\
21 & 439.59 & 352.67 & 624.7 & 3.391 & 0.125 \\
22 & 448.10 & 357.62 & 641.4 & 2.692 & 0.108 \\
\hline \hline
\end{tabular}

in all cases $P_{T} / P_{0}>2.5$. The maximum usable pressure ratio is that at which (1) there is no visible convection in the chamber and (2) $\rho_{1} / \rho_{0}$ continues to be a monotonically decreasing function of chamber height. ${ }^{8,9}$ It is not sufficient that $\rho_{1} / \rho_{0} \leqslant 1$ since high wall heat can easily overcome a slightly decreasing density gradient and cause convection. Rather, $\rho_{1} / \rho_{0} \ll 1$, and preferably $\leq 0.5 .^{41}$ The density gradients reported in Table II fulfill this requirement except, perhaps, at the lowest temperature (Experiment \#1) where convection is the least likely to invalidate experimental results. To further insure against convection we maintain the wall heat at the lowest level possible, i.e., at or slightly higher than the point at which menthol just begins to condense on the chamber walls. The maximum usable pressure ratio also depends on the molecular weights of the vapor and the carrier gas. The requirement that $\rho_{1} / \rho_{0} \ll 1$ can be translated into the condition ${ }^{4}$

$$
\frac{P_{T}}{P_{0}} \ll \frac{1-M_{v} / M_{g}}{1-T_{0} / T_{1}} \cong 152 .
$$

Our situation is optimal. Experiments are performed on a heavy vapor in a light carrier gas. Thus, the maximum usable pressure ratio is large $(\sim 152)$ for the menthol-helium system. The pressure ratios listed in Table II are all well below this upper limit.

The raw data in Table II is used as input for Eqs. (1) and (8) along with the thermophysical properties labeled " $\mathrm{e}$ " in Table I. From these we obtain the computer solutions of the mass and heat flux equations - the pressure and temperature profiles in the chamber as a func- 


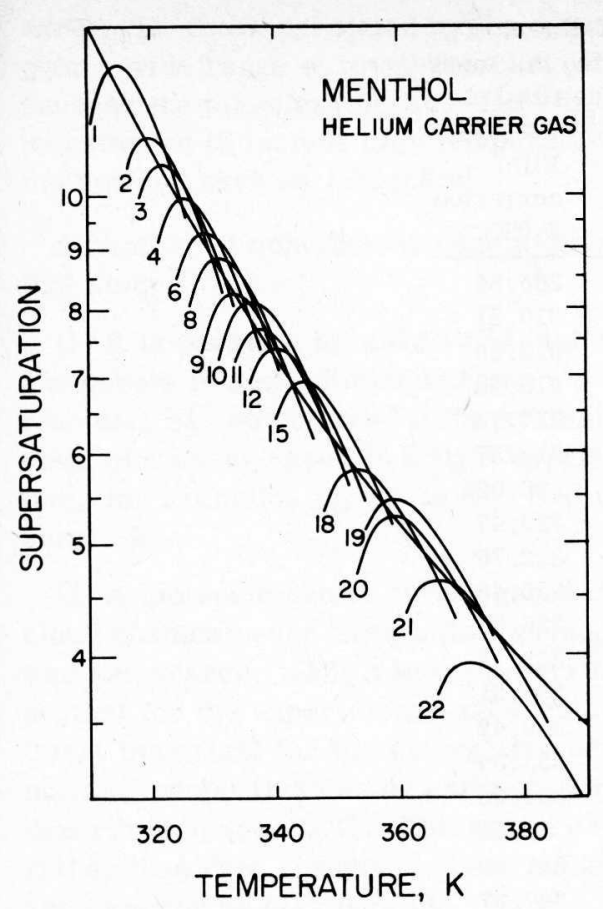

FIG. 2. Variation of the critical supersaturation of menthol vapor as a function of temperature. The solid curve is the critical supersaturation predicted by the classical BeckerDoering theory. The numbered curves are obtained from the raw data in Table II. The envelope of the numbered curves, which is not shown, is the experimental result.

tion of height, and an $L$ and a $q$ for each experiment. In Fig. 2 a portion of the supersaturation profile versus the corresponding temperatures is plotted for each experiment. The curves corresponding to Experiments $\# 5,7,13,14,16$, and 17 have been omitted since they can be superimposed on the curves already plotted. We obtain a critical supersaturation from each curve by realizing that, in any given experiment, the chamber is just barely nucleating when the data is taken. At only one thin horizontal plane in the chamber is the supersaturation large enough to equal the critical supersaturation. As a result, the experimental variation of critical supersaturation with temperature is the envelope to the individual curves. Each curve is tangent to the envelope at only one point, on the high temperature side of the peak $\left(S_{\mathrm{max}}\right) .^{3}$ The theoretical variation of critical supersaturation with temperature, computed from Eq. (13) and the properties in Table I, is the solid line in Fig. 2. Experimental critical supersaturations are, on the average, $6.6 \%$ higher than those predicted by the theory. As can be seen, the highest temperature point, \#22, falls below the theory curve. This anomalous behavior at high temperatures is in part an artifact of combining two sets of vapor pressure data to get one set of Antoine coefficients which covers the entire temperature range of the experiment. Above $110^{\circ} \mathrm{C}$, the Antoine coefficients listed in Table I give vapor pressures about $5 \%$ higher than the observed data from Stull's compilation. These high $P_{e}$ 's result in measured critical supersaturations 3.4\% lower for Experiment \#21 and 4.9\% lower for \# 22 than those obtained using the coefficients from Stull's data alone in the transport equations.

Since the real upper boundary of the chamber is the film surface, rather than the top plate surface, we compute the film correction using Eqs. (14) and (15) and the properties listed in Table I. In addition to these properties, three constants are required $\left(r, h\right.$, and $\left.\phi_{0}\right)$ which are characteristic of the chamber. In this case $r$ is taken as one-half the radius of the chamber, i.e., $r=3.57 \mathrm{~cm}$; the height at half-radius $h=2.68 \mathrm{~cm}$; and $\phi_{0}=0.017 \mathrm{rad}$, corresponding to the $1^{\circ}$ radial slope of the surface of the top plate. The remaining two unknowns in Eqs. (14) and (15), $L$ and $q$, are obtained directly from the solution of the mass and heat flux equations, respectively. There has been some controversy over the exact definition of $q$. Here, $q$ is the heat flux one would actually measure in the laboratory, the correct $q$ to be used in estimating the observed film correction.

The calculation of $t$ and $\Delta T$ is shown in Table III. As $\eta_{l}$ decreases exponentially with temperature, $L$ increases dramatically so that $t$ is about the same for all experiments. Therefore, since the temperature dependence of $\lambda_{1}$ is weak and nearly linear, ${ }^{42}$ the magnitude of $\Delta T$ is mainly determined by $q$. Large convective heat fluxes have previously been computed from the transport equations at low pressure ratios. ${ }^{9}$ The same effect is observed here. As can be seen from Table III, $q$ is very large for the highest temperature experiments \# 19-22, for which the pressure ratios are the lowest, ranging from 4.6-2.7, respectively. These large $q$ 's result in $\Delta T^{\prime}$ 's much larger than expected, i.e., $2.24^{\circ} \mathrm{K}$ for experiment \#22.

The computer solutions of the mass and heat flux equations are recalculated using the film corrected $T_{1}$ 's. The results are shown along with the theory curve in Fig. 3. As can be seen, critical supersaturations have been measured over a wide range of temperatures, from $41-100^{\circ} \mathrm{C}$. The agreement between experiment and theory is quite good over most of this range. For the 22 points the difference between the experimental envelope and the theoretical critical supersaturations averages $4.4 \%$.

Previous workers have concluded that the error in $S_{\max }$ due to neglecting $\Delta T$ is negligible. ${ }^{4,6,8}$ Figure 4 is designed to re-examine this conclusion. Some of the non-film corrected data taken directly from Fig. 2, curves 1, 6, 15, 20, and 22, are shown in Fig. 4. These are the upper curves labeled with primed numbers. For comparison the corresponding film corrected data from Fig. 3 is also plotted in Fig. 4 (unprimed) along with the theory curve. We can see that the film correction shifts the peak supersaturations downward considerably and that the downward trend becomes larger with increasing temperatures or decreasing pressure ratios. For all 22 points the average change in $S_{\max }$ produced by applying the film correction is $4.4 \%$. In fact, neglecting $\Delta T$ is a larger source of experimental error than calculating all the thermophysical properties (except the vapor pressure) required to solve the mass and heat flux equations. 
TABLE III. Calculation of the film correction for each experiment. Variables are as follows: $\boldsymbol{T}_{1}$, the temperature of the top plate; $\eta_{l}$, the liquid viscosity; $L$, the reduced mass flux; $t$, the thickness of the condensed film; $q$, the reduced heat flux; and $\Delta T$, the temperature drop across the film.

\begin{tabular}{|c|c|c|c|c|c|c|c|}
\hline $\begin{array}{l}\text { Experiment } \\
\text { number }\end{array}$ & $\begin{array}{l}\text { Raw data } \\
T_{1}\left({ }^{\circ} \mathrm{K}\right)\end{array}$ & $\eta_{l}(\mathrm{cP})$ & $\begin{array}{l}L \times 10^{6} \\
(\mathrm{~mole} / \mathrm{cm} \mathrm{sec})\end{array}$ & $t(\mathrm{~mm})$ & $\begin{array}{l}q \times 10^{2} \\
(\mathrm{cal} / \mathrm{cm} \mathrm{sec})\end{array}$ & $\Delta T\left({ }^{\circ} \mathrm{K}\right)$ & $\begin{array}{l}\text { Film } \\
\text { corrected } \\
T_{1}\left({ }^{\circ} \mathrm{K}\right) \\
\end{array}$ \\
\hline 1 & 305.07 & 31.274 & 0.252 & 0.122 & 2.872 & 0.49 & 305.56 \\
\hline 2 & 309.71 & 22.245 & 0.342 & 0.121 & 2. 988 & 0.50 & 310.21 \\
\hline 3 & 311.68 & 19.295 & 0.393 & 0.121 & 3.050 & 0.52 & 312.20 \\
\hline 4 & 314.95 & 15.315 & 0.554 & 0.126 & 3.226 & 0.57 & 315.52 \\
\hline 5 & 317.20 & 13.098 & 0.534 & 0.118 & 3.231 & 0.54 & 317.74 \\
\hline 6 & 317.99 & 12.407 & 0.645 & 0.123 & 3.360 & 0.59 & 318.57 \\
\hline 7 & 319.45 & 11.220 & 0.625 & 0.118 & 3.380 & 0.57 & 320.02 \\
\hline 8 & 319.92 & 10.867 & 0.593 & 0.115 & 3.353 & 0.55 & 320.47 \\
\hline 9 & 322.16 & 9.348 & 0.772 & 0.120 & 3.516 & 0.60 & 322.76 \\
\hline 10 & 324.82 & 7.835 & 0.835 & 0.116 & 3. 671 & 0.61 & 325.43 \\
\hline 11 & 326.23 & 7.144 & 0.898 & 0.115 & 3.727 & 0.62 & 326.84 \\
\hline 12 & 328.50 & 6.166 & 0.962 & 0.112 & 3.843 & 0.62 & 329.12 \\
\hline 13 & 329.63 & 5.736 & 1.112 & 0.115 & 4.083 & 0.68 & 330.31 \\
\hline 14 & 329.75 & 5.692 & 1. 104 & 0.115 & 4.077 & 0.68 & 330.42 \\
\hline 15 & 332.62 & 4.996 & 1. 297 & 0.116 & 4. 322 & 0.73 & 333.34 \\
\hline 16 & 333.60 & 4.789 & 1. 367 & 0.116 & 4.484 & 0.76 & 334.36 \\
\hline 17 & 333.69 & 4.771 & 1. 519 & 0.121 & 4.688 & 0.82 & 334.51 \\
\hline 18 & 340.40 & 3.595 & 1.607 & 0.112 & 4.822 & 0.79 & 341.20 \\
\hline 19 & 346.23 & 2.838 & 2. 511 & 0.121 & 6.413 & 1.15 & 347.37 \\
\hline 20 & 346.33 & 2.826 & 2.759 & 0.124 & 6.814 & 1.25 & 347.59 \\
\hline 21 & 352.67 & 2.205 & 3.661 & 0.126 & 8.618 & 1.62 & 354.29 \\
\hline 22 & 357.62 & 1.827 & 4. 958 & 0.132 & 11.333 & 2.24 & 359.86 \\
\hline
\end{tabular}

\section{SUMMARY AND CONCLUSIONS}

In summary, the equations and properties required to analyze the raw cloud chamber data, compute the theory, and calculate the film correction are discussed in Sec.

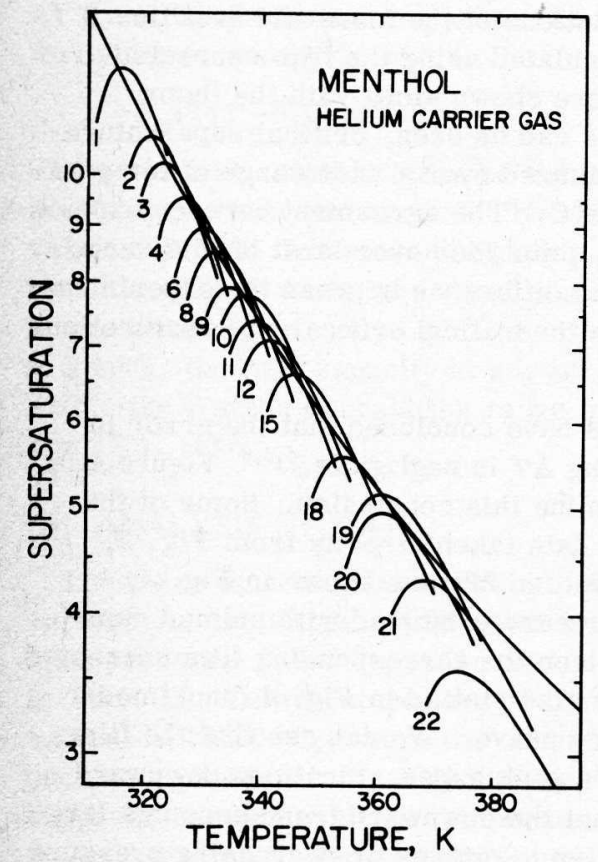

FIG. 3. Variation of the critical supersaturation of menthol vapor as a function of temperature. The solid curve is the critical supersaturation predicted by the classical BeckerDoering theory. The numbered curves are obtained using the film corrected $T_{1}$ 's presented in Table III. The envelope of the numbered curves, which is not shown, is the experimental result.
II. In Sec. III the raw data is presented and shown to satisfy the pressure ratio and density ratio stability criteria. Also, in Sec. III the data is analyzed, i.e., the supersaturation and temperature profiles are calculated. Measured critical supersaturations are found to

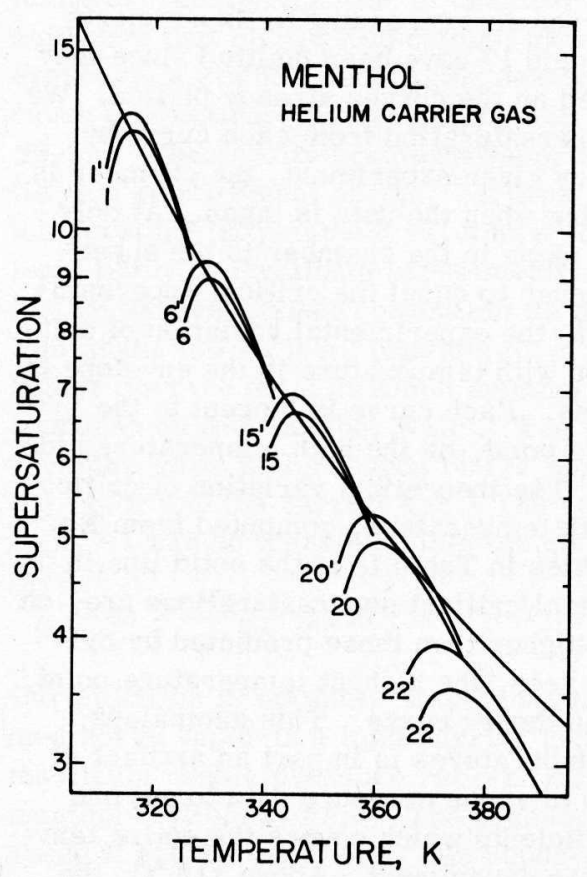

FIG. 4. Comparison of raw data taken directly from Fig. 2 (upper curves labeled with primed numbers) and the corresponding film corrected data from Fig. 3 (lower unprimed curves). The solid curve is the variation of the critical supersaturation with temperature predicted by the classical BeckerDoering theory. 
agree with those predicted by the theory to within $5 \%$ over a wide range of temperatures. On the basis of this success the classical Becker-Doering theory can indeed be extended to include high temperatures and unique molecules, such as $l$-menthol.

A number of conclusions can be drawn from the menthol study:

(1) It is possible to make valid high temperature measurements in a small cloud chamber. Such convectionfree data can be obtained if the system of interest consists of a heavy vapor in a light carrier gas. For menthol, for example, $M_{v} / M_{g}$ in Eq. (19) is large, i.e., about 39 .

(2) A molecule can be investigated in the diffusion cloud chamber even though its thermophysical properties are scarce. Only vapor pressure data (most important for the experiment) and surface tension data (most important for the theory) are necessary. The remaining properties can be estimated by the methods described in Sec. II. C. The use of estimated values, rather than data, results in peak supersaturations which are in error by less than $1 \%$.

(3) The magnitude of the film correction is largely determined by $q$, which becomes large at low pressure ratios, or high temperatures.

(4) The film correction is not negligible. For menthol, for example, adding $\Delta T$ to the top plate temperatures alters the peak supersaturations by $4.4 \%$. Therefore, neglecting $\Delta T$ is a larger source of experimental error than estimating all the properties (except the vapor pressure) needed to solve the mass and heat flux equations.

Finally, the results of this study suggest several areas for future investigation. The vapor pressure expression for menthol was obtained by merging two sets of data, each valid over a different temperature range. The average error in the Antoine equation in Table I, which fits the combined data, is $4.2 \%$. Since the vapor pressure is critical to the experimental results, one set of self-consistent vapor pressure data, measured over the range $30-175^{\circ} \mathrm{C}$, is needed.

For the non-film corrected data shown in Fig. 2 the experimental envelope is $6.6 \%$ higher than the critical supersaturations predicted by the Becker-Doering theory. This suggests that menthol may be somewhat associated in the vapor. ${ }^{43,44}$ Given a more accurate vapor pressure expression, the degree of association can be determined.

\section{ACKNOWLEDGMENTS}

This research was supported by Grant MPS74-22922 (CHE77-08537) from the National Science Foundation, Washington, D.C.

We thank Roger Chang, Rockwell International Corporation, for telling us about the existence of unpublished vapor pressure and surface tension data for menthol and Joseph Katz for making his PLOT program available.
We are grateful to Erik Olsen for his expertise in machining the cloud chamber plates and baths.

\section{APPENDIX. ESTIMATION METHODS FOR VAPOR VISCOSITY}

The refined kinetic theory of Chapman and Enskog is recommended for estimating gas viscosities. The Chapman-Enskog equation (CEE), which includes interactions between colliding molecules, is ${ }^{16}$

$$
\eta=26.69 \times 10^{-3}\left[\frac{(M T)^{1 / 2}}{\sigma^{2} \Omega}\right],
$$

where $\eta$ is the gas viscosity (cP), $\sigma$ is the collision diameter, and $\Omega$ is the collision integral. For nonpolar molecules $\Omega$ is evaluated using the Lennard-Jones potential, with parameters $\epsilon / k$ and $\sigma$; for polar molecules with angle-dependent forces the Stockmayer potential function is more reasonable. The Stockmayer parameter $\delta$ can be used to distinguish between polar and nonpolar molecules. It allows for a dipole moment $\mu_{p}$ and is defined as

$$
\delta=\mu_{p}^{2} / 2 \in \sigma^{3} \text {, }
$$

where $\epsilon$ is the characteristic energy. If $\delta<0.1$, a molecule is considered nonpolar, ${ }^{45}$ and $\Omega$ in $\mathrm{Eq}$. (A1) is taken as the Lennard-Jones collision integral. This is the case for menthol for which $\delta=0.07$. The three methods outlined below are essentially based on the Chapman-Enskog theory [Eq. (A1)].

CEE and boiling point constants. $\epsilon / k$ (the force constant) and $\sigma$ are estimated for menthol according to Spenser et al. ${ }^{46}$ as

$$
\begin{aligned}
& \epsilon / k=1.15 T_{b}, \\
& \sigma=1.166\left(V_{b}\right)^{1 / 3},
\end{aligned}
$$

where $V_{b}$ is the molar volume of the liquid at $T_{b}$ and $k$ is the Boltzmann constant. $\Omega$ has been calculated and tabulated as a function of $k T / \epsilon$ by Hirschfelder, Curtiss, and Bird. ${ }^{47}$

CEE and critical constants. $\epsilon / k$ and $\sigma$ are estimated from the critical constants by ${ }^{46}$

$$
\begin{aligned}
& \epsilon / k=0.77 T_{c}, \\
& \sigma=0.841\left(V_{c}\right)^{1 / 3},
\end{aligned}
$$

where $V_{c}$ is the critical volume. $T_{c}$ and $V_{c}$ are unknown for menthol, but we calculate them using Lydersen's method of structural contributions. ${ }^{18}$

Bromley and Wilke method. This method is similar to the one above in that $\epsilon / k$ and $\sigma$ have been replaced by the critical constants. The result is the BromleyWilke equation ${ }^{48}$

$$
\eta=\frac{\left(3.33 \times 10^{-3}\right)\left(M T_{c}\right)^{1 / 2} f_{1}\left(1.33 T_{r}\right)}{V_{c}^{2 / 3}}
$$

Here, $f_{1}\left(1.33 T_{r}\right)$ is a tabulated function in which $k T / \epsilon$ is approximated by $1.33 T_{r}$. With the use of estimated critical properties the average error in Eq. (A5) is about $8 \%{ }^{48}$ 
${ }^{1}$ J. P. Franck and H. G. Hertz, Z. Phys. 143, 559 (1956).

${ }^{2}$ H. G. Hertz, Z. Elektrochem. 60, 1196 (1956).

${ }^{3}$ J. L. Katz and B. J. Ostermier, J. Chem. Phys. 47, 478 (1967):

${ }^{4} J$. L. Katz, J. Chem. Phys. 52, 4733 (1970).

${ }^{5}$ H. L. Jaeger, E. J. Willson, P. G. Hill, and K. C. Russell,

J. Chem. Phys. 51, 5380 (1969).

${ }^{6}$ R. H. Heist and H. Reiss, J. Chem. Phys. 59, 665 (1973).

${ }^{7}$ D. B. Dawson, E. J. Willson, P. G. Hill, and K. C. Russell, J. Chem. Phys. 51, 5389 (1969).

${ }^{8}$ J. L. Katz, C. J. Scoppa II, N. G. Kumar, and P. Mirabel, J. Chem. Phys. 62, 448 (1975).

${ }^{9} J$. L. Katz, P. Mirabel, C. J. Scoppa II, and T. L. Virkler, J. Chem. Phys. 65, 382 (1976).

${ }^{10}$ H. R. Nace and R. H. Nealey, J. Am. Chem. Soc. 88,65 (1966).

11 J. Sanchez, Diss. Abstr. B 28, 116 (1967).

${ }^{12} \mathrm{~J}$. Emerson, Matheson Coleman and Bell Manufacturing Chemists (private communication, 1976).

${ }^{13} \mathrm{E}$. Guenther, The Essential Oils (Van Nostrand, Toronto, 1949), Vol. 3, p. 597.

${ }^{14}$ W. Licht, Jr. and D. G. Stechert, J. Phys. Chem. 48, 23 (1944).

${ }^{15}$ D. N. Rihani and L. K. Doraiswamy, Ind. Eng. Chem. Fund. 4, 17 (1965).

${ }^{16} \mathrm{~J}$. O. Hirschfelder, C. F. Curtiss, and R. B. Bird, Molecular Theory of Gases and Liquids (Wiley, New York, 1954), Chap. 8.

${ }^{17}$ K. M. Watson, Ind. Eng. Chem. 35, 398 (1943).

${ }^{18}$ A. L. Lydersen, "Estimation of Critical Properties of Organic Compounds" in University of Wisconsin, College of Engineering, Engineering Experiment Station Report 3 (1955).

${ }^{19} \mathrm{~L}$. Riedel, Chem. Ing. Tech. 26, 679 (1954).

${ }^{20}$ For vapor pressures below $10 \mathrm{torr}$, no reliable estimation methods are available (see Ref. 23, p. 196). Above 10 torr, vapor pressures can be calculated by an integrated form of the Clausius-Clapeyron equation which requires the enthalpy of vaporization at the normal boiling point and both the liquid and vapor heat capacities. ${ }^{9}$ However, using calculated values for these three properties increases the error in the integrated vapor pressure expression to at least $8 \%$. The effect of an uncertainty of this magnitude on the supersaturation profile in the chamber has been investigated for menthol. We find that changing $P_{e}$ by $10 \%$ changes the peak supersaturations by as much as $6.3 \%$.

${ }^{21} \mathrm{D}$. R. Stull, Ind. Eng. Chem. 39, 517 (1947).

${ }^{22} \mathrm{G}$. Ervin, Atomics International Division, Rockwell International Corporation (private communication, 1977).

${ }^{23}$ R. C. Reid, J. M. Prausnitz, and T. K. Sherwood, The
Properties of Gases and Liquids (McGraw-Hill, New York, 1977), 3rd edition, p. 508.

${ }^{24}$ A. L. Lindsay and L. A. Bromley, Ind. Eng. Chem. 42, 1508 (1950).

${ }^{25}$ E. N. Fuller, P. D. Schettler, and J. C. Giddings, Ind. Eng. Chem. 58, 19 (1966).

${ }^{26}$ Viscosity, Thermophysical Properties of Matter (IFI/Plenum, New York, 1970), Vol. 2, p. 18.

${ }^{27}$ Thermal Conductivity: Nonmetallic Liquids and Gases, Thermophysical Properties of Matter (IFI/Plenum, New York, 1970), Vol. 3, p. 33.

${ }^{28} \mathrm{R}$. Becker and W. Doering, Ann. Phys. (Leipzig) 24, 719 (1935).

${ }^{29} \mathrm{~J}$. Frenkel, Kinetic Theory of Liquids (Oxford University, London, 1946), Chap. 7.

${ }^{30} \mathrm{~J}$. Timmermans, Physico-Chemical Constants of Pure Organic Compounds (Elsevier, New York, 1950), Vol. 1, p.496.

${ }^{31}$ Beilsteins Handbuch der Organischen Chemie (Springer, Berlin, 1965), 4th edition, Supplement 3, Vol. 6, Pt. 1, p. 133.

${ }^{32} \mathrm{R}$. Chang, Rockwell International Corporation (private communication, 1977). P. J. Dynes measured these surface tensions for menthol using the ring method.

${ }^{33}$ Reference 22 , p. 437.

${ }^{34}$ International Critical Tables (McGraw-Hill, New York, 1930), Vol. 7, p. 221.

${ }^{35}$ W. Hückel and H. -W. Wunsch, J. Prakt. Chem. 142, 15 (1935).

${ }^{36}$ L. A. Robbins and C. L. Kingrea, Hydrocarbon Process. Petrol. Refiner 41, 133 (1962).

${ }^{37}$ D. H. Everett, J. Chem. Soc. 1960, 2566.

${ }^{38}$ W. M. Chow and J. A. Bright, Jr., Chem. Eng. Prog. 49, 175 (1953).

${ }^{39}$ O. A. Hougen and K. M. Watson, Chemical Process Principles (Wiley, New York, 1950), p. 504.

${ }^{40}$ A. I. Johnson and C.-J. Huang, Can. J. Technol. 33, 421 (1955).

${ }^{41} \mathrm{~J}$. L. Katz, Clarkson College of Technology (private communication, 1977).

${ }^{42}$ Reference 22 , p. 516.

${ }^{43}$ J. L. Katz, H. Saltsburg, and H. Reiss, J. Colloid Interface Sci. 21, 560 (1966).

${ }^{44}$ R. H. Heist, K. M. Colling, and C. S. DuPuis, J. Chem. Phys. 65, 5147 (1976).

${ }^{45}$ Reference 22, p. 402 .

${ }^{46}$ H. B. Spencer, J. M. Toguri, and J. A. Kurtis, Can. J. Chem. 47, 2197 (1969).

${ }^{47}$ Reference 16, p. 1126.

${ }^{48}$ L. A. Bromley and C. R. Wilke, Ind. Eng. Chem. 43, 1641 (1951). 